\title{
The Geometry Optimization of Argon Atom Clusters Using Differential Evolution Algorithm
}

\author{
Yongxiang Zhao, Shengwu Xiong, and Ning Xu \\ School of Computer Science and Technology, Wuhan University of Technology, \\ Wuhan 430070, China \\ zhaosanhe@263.net, xiongsw@mail.whut.edu.cn
}

\begin{abstract}
Recently atomic cluster structures have been intensively studied because of their importance in physics, chemistry and material science. However, finding the lowest energy structure, which is the most stable configuration, is NP-hard. Differential Evolution (DE) algorithm is a new heuristic approach which mainly has three advantages: finding the true global minimum regardless of the initial parameter values, fast convergence, and using few control parameters. In this paper we describe a new search method that uses differential evolution (DE) algorithm to optimize the geometry of small argon atom clusters. Experimental results show that the exact global optimal configuration of argon clusters with atom number $\mathrm{N} \leq 16$ can be found in a reasonable computing time, and approximate optimization can also be obtained for clusters with $\mathrm{N}=30$. From their $3-\mathrm{D}$ geometry structures, we can see that their optimal energy structures are highly symmetrical.
\end{abstract}

Keywords: Argon Atom Cluster, Structure Optimization, Genetic Algorithm, Differential Evolution.

\section{Introduction}

Recently atomic cluster structures have been intensively studied because of their importance in physics, chemistry and material science. One method of solving this optimization problem is to explore the potential energy surface (PES) composed of all possible cluster conformations. Unfortunately, as the cluster size increases, so does the number of degrees of freedom in the placement of the atoms. This characteristic produces a PES where the number of local optima grows exponentially with the cluster size [1]. Determining the ground-state energy level, which is the most energetically stable level, is extremely difficult. Wille and Vennik [2] proved this problem is NP-hard for homo-nuclear clusters (i.e., clusters with only one type of atom) and Greenwood [3] later proved the same thing for hetero-nuclear clusters.

Hence, heuristic search techniques are widely used in this area, such as Genetic algorithms [4,5], Simulated Annealing [6].

Differential Evolution (DE) algorithm [7,8] is a new heuristic approach which mainly has three advantages: finding the true global minimum regardless of the initial parameter values, fast convergence, and using few control parameters. In this paper 
we describe a new search method that uses differential evolution (DE) algorithm to optimize the geometry of small argon atom clusters. Experimental results show that the exact global optimal configuration of argon clusters with atom number $\mathrm{N} \leq 16$ can be found in a reasonable computing time, and approximate optimization can also be obtained for clusters with $\mathrm{N}=30$.

The paper is organized as follows. An overview of atomic clusters is provided in Section 2. Section 3 reviews the DE approach and shows how it is used to search for low energy conformations. Section 4 presents some experiments conducted with small clusters of argon atoms. Finally, Section5 concludes the paper with summary and future research directions.

\section{Homo-nuclear Clusters and Potential Energy Functions}

The objective is to find the lowest energy conformation because it is the most stable conformation. In this work, we approximate the total energy as the sum of all the pairwise interactions between atoms:

$$
E=\sum_{i=1}^{N-1} \sum_{j=i+1}^{N} v\left(r_{i j}\right)
$$

Where $r_{i j}$ is the Euclidean distance between atoms $i$ and $j$, and $v\left(r_{i j}\right)$ is the pairwise potential energy function.

A commonly used pairwise potential energy function for (1) is the Lennard-Jones potential function [4]:

$$
\begin{gathered}
v\left(r_{i j}\right)=4.0 \varepsilon\left[\left(\frac{\sigma}{r_{i j}}\right)^{12}-\left(\frac{\sigma}{r_{i j}}\right)^{6}\right] \\
r_{i j}=\sqrt{\left(x_{i}-x_{j}\right)^{2}+\left(y_{i}-y_{j}\right)^{2}+\left(z_{i}-z_{j}\right)^{2}}
\end{gathered}
$$

where $\varepsilon=1, \sigma=3.36 \AA, x_{i}, y_{i}, z_{i} \in[-1 / 2 \cdot \sqrt[3]{6 N} \cdot \sigma, 1 / 2 \cdot \sqrt[3]{6 N} \cdot \sigma]$.

\section{Using DE in Cluster Searches}

The DE algorithm is a population based algorithm like genetic algorithms using the similar operators: crossover, mutation and selection [7]. The main difference in constructing better solutions is that genetic algorithms rely on crossover while DE relies on mutation operation. This main operation is based on the differences of randomly sampled pairs of solutions in the population.

The algorithm uses mutation operation as a search mechanism and selection operation to direct the search toward the prospective regions in the search space. The DE algorithm also uses a non-uniform crossover that can take child vector parameters from one parent more often than it does from others. By using the components of the existing population members to construct trial vectors, the recombination (crossover) operator efficiently uses information about successful combinations, enabling the search for a better solution space. 
An optimization task consisting of $\mathrm{D}$ parameters can be represented by a D-dimensional vector. In DE, a population of NP solution vectors is randomly created at the start. This population is successfully improved by applying mutation, crossover and selection operators. The details of the mutation, crossover and selection operators were described in literature [7].

\section{Experimental Results}

These experiments were all executed under Microsoft Windows Server 2003 with 3.00GHz of Intel(R) Pentium(R) 4 CPU and $512 \mathrm{MB}$ of RAM. Each experiment was executed 10 times, and the parameters of the DE algorithm [7] in the experiments were as follows:

Dimensions $\mathrm{D}=3 \mathrm{~N}$, where $\mathrm{N}$ is argon atom number, the number of population $\mathrm{NP}=30$, the scale factor $\mathrm{F}=0.35$, the crossover rate $\mathrm{CR}=0.2$, the maximum generation is 100000, and we use the strategy DE/rand-to-best/1/exp.

The optimized energy results for argon atom clusters are shown in Table 1. Experimental results show that the exact global optimal configuration of argon clusters with atom number $\mathrm{N} \leq 16$ can be found in a reasonable computing time, and approximate optimization can also be obtained for clusters with $\mathrm{N}=30$.

The optimized geometry structures for argon atom clusters $(\mathrm{N}=12,14,16$ and 30) are shown in Fig. 1. From the pictures, we can see that their geometry structures are highly symmetrical.

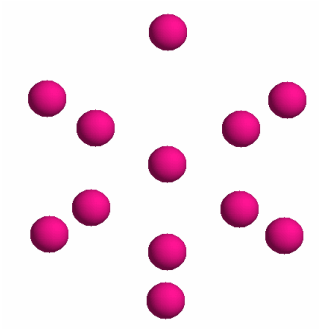

(a) atom number $\mathrm{N}=12$

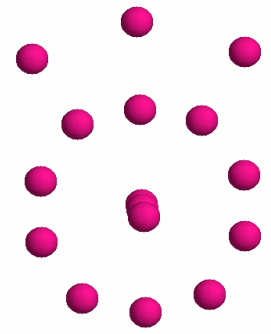

(c) atom number $\mathrm{N}=16$

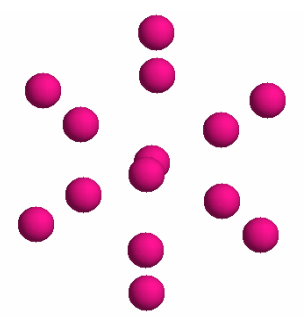

(b) atom number $\mathrm{N}=14$

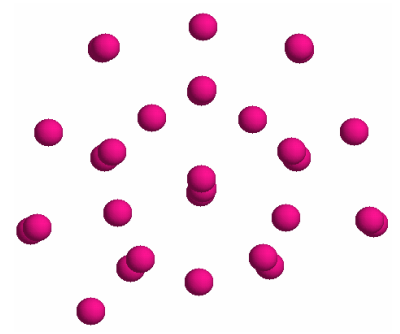

(d) atom number $\mathrm{N}=30$

Fig. 1. The structure of argon atom clusters optimized by DE algorithm. Drawn with RasMol [9]. 
Table 1. The optimized energy by DE and its comparison with literature value

\begin{tabular}{ccc}
\hline Atom number N & Energy in Literature[4] & Energy in DE algorithm \\
\hline 12 & -37.97 & -37.968 \\
13 & -44.33 & -44.330 \\
14 & -47.85 & -47.845 \\
15 & -52.32 & -52.320 \\
16 & -56.82 & -56.816 \\
30 & -128.29 & -127.286 \\
\hline
\end{tabular}

\section{Summaries and Future Work}

In this paper we use differential evolution algorithm to find global minimum energy structure of small argon clusters. Experimental results show that the exact global optimal configuration of argon clusters with atom number $\mathrm{N} \leq 16$ can be found in a reasonable computing time, and approximate optimization can also be obtained for clusters with $\mathrm{N}=30$. From their $3-\mathrm{D}$ geometry structures, we can see that their optimal energy structures are highly symmetrical.

For future work, we will use the proposed DE method in other optimization areas, such as $\mathrm{Si}$ atomic clusters and protein 3-D structure prediction.

Acknowledgments. This work was in part supported by NSFC (Grant No. 60572015) and 973 Pre-research Project (Grant No. 2004CCA02500).

\section{References}

1. Berry, R.: Potential surfaces and dynamics: what clusters tell us. Chem. Rev., Vol. 93, (1993) 2379-2394

2. Wille, L., Vennik, J.: Computational complexity of the ground-state determination of atomic clusters. J. Phys. A, Vol. 18, (1985) L419-L422

3. Greenwood, G.: Revisiting the complexity of finding globally minimum energy configurations in atomic clusters. Z Phys. Chem., Vol. 211, (1999) 105-114

4. JIANG, H.Y.: The Geometry Optimization of Argon Atom Clusters Using a Parallel Ge-netic Algorithm, Computers and Applied Chemistry, Vol. 19, (2002) 9-12 (In Chinese)

5. XIA, B.Y.: The Optimization of the Argon Atom Cluster Structure Using a Modified Genetic Algorithm, Computers and Applied Chemistry, Vol. 18, (2001) 139-142 (In Chinese)

6. Stillinger, F., Weber, T.: Computer simulation of local order in condensed phases of argon. Phys. Rev.B, Vol. 31, (1985) 5262-5268

7. Storn, R., Price, K.: Differential evolution: a simple and efficient adaptive scheme for global optimization over continuous spaces. Technical Report TR-95-012, International Computer Science Institute, Vol. 8, (1995) 22-23

8. Abbass, H.A., Sarker, R., Newton, C.: PDE: A pareto-frontier differential evolution approach for multi-objective optimization problems. CEC2001 Proceedings, (2001) 971-978

9. Sayle, R., Milner-White, E.J.: RasMol: Biomolecular Graphics for All, Trends Biochem. Sci. Vol. 20, (1995) 374-376 